\title{
INVESTIGATION OF SECURE VEKIT WEB INTERFACE FOR PREVENTING ENVIRONMENT POLLUTION
}

\author{
Kumar BALAJI ${ }^{1^{*}}$, Muthusamy SELVAM ${ }^{2}$ \\ ${ }^{1}$ Dept of Electronics and Communication, SSM College of Engineering, India \\ ${ }^{2}$ Dept of Computer Science Engineering, Excel Engineering College, India
}

Received 14 November 2018; revised 22 December 2018; accepted 1 March 2019;

first published online 1 September 2020

\begin{abstract}
This paper describes a new system - Vehicle Kit (VEKIT), which uses a secure web-based interface for the authentication and monitoring of Vehicular Ad-Hoc Network (VANET) infrastructure and for preventing environment pollution. This paper presents the hazards caused by the usage of bags made of plastic material and proposes the design of VEKIT, which is to be installed in the vehicles for reducing the usage of such carry bags up to $75 \%$. The survey results prove that the proposed VEKIT approach is very much relevant to solve the pollution problem faced by various countries. This novel application adds value to the future VANET projects by helping the countries to prevent pollution. The web interface of VEKIT presented in the paper can also be used to monitor the vehicles for their authenticity. A secure architecture is proposed for the usage of VANET in transport related projects.
\end{abstract}

Keywords: carry bags, environment pollution, RFID, VANET, VEKIT prototype, Wi-Fi, vehicle.

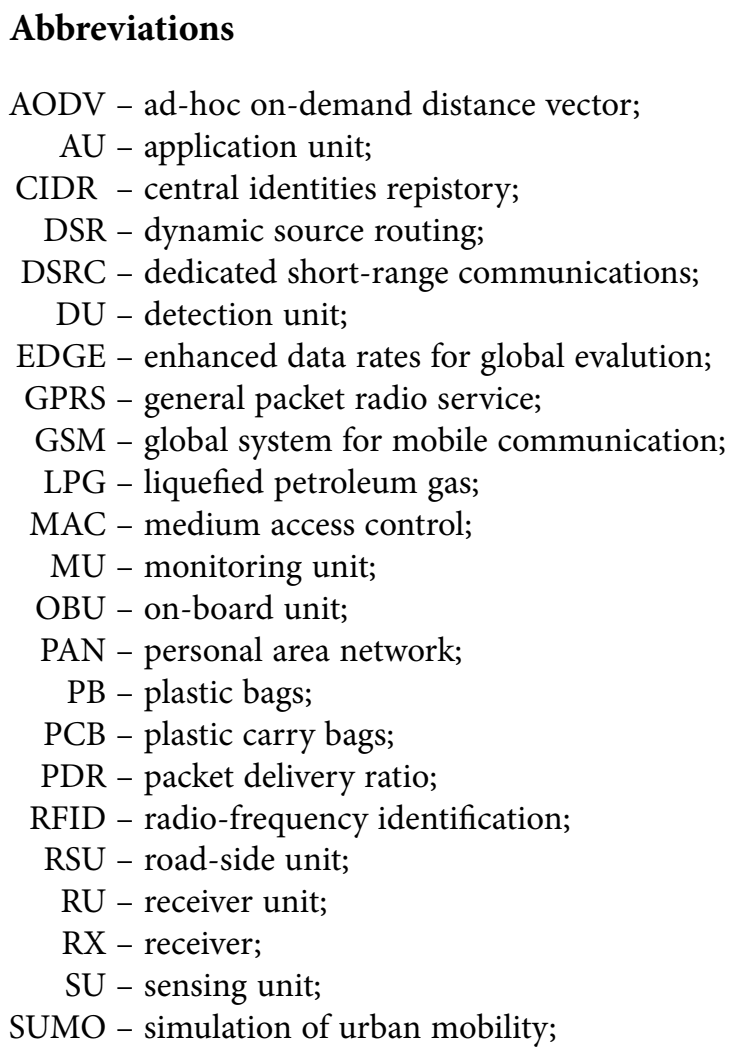

\author{
TU - trigger unit; \\ TX - transmitter; \\ UIDAI - unique identification authority of India; \\ V2RSU - vehicle-to-RSU; \\ V2V - vehicle-to-vehicle; \\ VANET - vehicular ad-hoc network; \\ VEKIT - vehicle kit; \\ Wi-Fi - wireless fidelity; \\ WLAN - wireless local area network.
}

\section{Introduction}

VANET, consisting of a network of vehicles moving at a high speed, equipped with devices that enables the vehicle to communicate with other vehicles and the RSU. VEKIT is a novel system proposed and designed to fit in the VANET projects in transport sector to check the authenticity of the participating vehicles and as a value addition to prevent the pollution of environment caused by the usage of PCB. It is a well known and widely proved fact that the usage of plastic material is very hazardous to the environment, wild life, mankind and the earth as a whole. The environment activists and the Governments are tak-

${ }^{*}$ Corresponding author. E-mail: balajiabboy@gmail.com 
ing enough measures to reduce the usage of plastic material. But the efforts are not working to the expected levels. Out of the different kind of the plastic materials that spoil the environment, the material used for making carry bags, consisting of polymers less than $30 \mu \mathrm{m}$ are causing major problem. These carry bags constitute a greater percentage of the total plastic material consumed. The goal is to reduce those single use $\mathrm{PCB}$, which are very popular and widely used in developing countries. VEKIT is a kit designed to be implemented in the vehicles, which can help to reduce the usage of such PCB up to $75 \%$, which is evident from our survey results. The research is based on the Vehicular network project applications related to transport domain and the VEKIT based methodology adds value to such projects. The Aadhaar Based Web interface is used to verify the authenticity of the VEKIT and its functionality. This paper explains the related previous applications of VANET projects, hazards of PCB, our survey, VANET overview, VEKIT prototype, Aadhaar based web interface, design and implementation technologies behind VEKIT project.

\section{Related previous works}

This section gives a review of various VANET related applications and use cases reported in the literature. In research by Al-Sultan et al. (2014) the VANET applications are classified into two broad categories namely: (1) comfort/entertainment, (2) safety applications. VANET applications are used for different scenarios with a particular usage. According to the detailed survey presented by Karagiannis et al. (2011), the different types of applications of VANETs can be broadly classified into road safety applications, traffic management applications and information broadcasting applications. Examples of active road safety applications include intersection collision warning, lane change assistance, rear and end collision warning, speed management and co-operative navigation are examples of traffic management applications. Co-operative local services and global Internet services are two classes of Infotainment applications.

The major intelligent transportation system projects carried out in US like IntelliDrive ${ }^{\mathrm{TM}}$ (Cronin 2015) enable secure wireless communication among vehicles and had 20 usecases during initial deployment. Vehicle safety communications (Ahmed-Zaid et al. 2011) implemented traffic safety applications like forward collision warning, curve speed warning etc. SafeTrip-21 is a VANET project that delivered assistance to the drivers to reach their destinations safely (Chandler et al. 2010).

Apart from very few applications and use cases of VANET, all other fall into one of the two or three classes of applications of VANET as mentioned above in the works by Al-Sultan et al. (2014) and Karagiannis et al. (2011). The VEKIT work presented in this paper provides two new class of applications to VANET deployment in: (1) preventing environment pollution by reducing the usage of plastic based carry bags, (2) automatic inspection of vehicles. So the projects mentioned above can include VEKIT as an additional feature to reduce the pollution problem. The pollution hazards of PCB and our VEKIT methodology to reduce the pollution are discussed in the following sections.

\section{Pollution hazards of plastic}

The polymer that makes up plastic never dies (Attinger 2011). The polymer cannot be destroyed completely but just break into smaller molecules. The research demonstrates that, except for a little sum of plastic that has been burned, all of plastic produced on the planet throughout the previous 50 years or so still remains. It's some place in the environment. This doesn't simply mean plastic in the bags - it implies all of plastic that is bought and discarded, from the water bottle, juice container, wrap on the new bit of electronic gadgets, etc.

Normally while going for shopping, people are having the habit of demanding for a single use bags made of plastic to carry their items purchased. These bags are made of chemicals that are almost indestructible (Attinger 2011). This habit is causing a serious damage to the environment. This paper gives a way, which is presented in Section 7, to reduce our habit of using a single use bags made of plastic. Although the single use $\mathrm{PB}$ used for carrying the items purchased while shopping can be used for multiple times, people tend to throw it out. This is the reason for the pollution as the chemical polyethylene used for making such bags is not destructible completely. The problem is very serious as we have been producing such bags for about 50 years where estimates predict that the total production would be over a billion tons of plastic.

\section{Problems posed by PCB}

The usage of PB create unclean environment as the disposed bags blocks the drainage (Kalpana 2010). The recycling process also adds the pollution as the colors and the synthetic substances taint the soil and the sub-soil water. The recycling units, which are not having facility to completely recycle the plastic may produce toxic gases leads to air pollution. The food particles, which remain in the PCB are swallowed by animals. These bags also arrest the ground water sources. Apart from these hazards the chemicals used to manufacture the plastic also create health problems.

\subsection{Health hazards for mankind}

Plastics are not intrinsically toxic or harmful (Kalpana 2010). The additives and colorants used to manufacture PCB and metals like cadmium and lead used in the manufacturing process creates harm to heart and brain.

\subsection{Problems for wildlife}

PB used for shopping thrown in the open environment cause a threat to wildlife (KWT 2016). Reports show that marine animals and creatures mistake these PCB for prey 
species and consume them. The PB floating in water are consumed by the marine animals and birds. Every year millions of marine animals are affected by plastic waste and single use carry bags thrown in the environment.

\section{Reason for focusing plastic in the shopping bags}

Our research is focused on reducing plastic consumption in the carry bag usage. Although plastic is used in more of products, there are concrete reasons to reduce the usage of PCB. The PCB designed for single use are disposed in large numbers and create pollution problems as mentioned above. The pollution problem is the critical problem to be analyzed in bringing any policy decisions related to shopping bags (EA 2006). The VEKIT concentrates on developing a practice of using reusable bags available in the VEKIT and a monitoring procedure to verify the condition of the VEKIT by the authorities. In this context our research findings and recommendations prove to be very useful for environment protection.

\section{Our research methodology}

This research work is based on the extensive data obtained from the survey conducted by collecting forms from customers in various shops on PCB usage. Our approach is based on finding the reason for the need for PCB and finding alternative to the need. We made a survey in various shops and the result of the survey is shown in Figure 1. The focus of the survey is to categorize the customers coming to various shops on the basis of the transportation they use.

The different categories showed in the Figure 1 represent the shops under consideration. The graph shows the number of buyers came by different mode of transport in each category of the shops. As we expected the, a huge percentage of the buyers used either two wheelers or four wheelers. This percentage would be even more in other developed countries.

\section{VEKIT policy based approach}

The first phase of our research survey was to find the percentage of customers using their two wheelers or four wheelers for shopping. The second phase of our research is to find the percentage of the customers accepting the policy. The policy, which we propose requires an extra fitting (VEKIT) in the two wheeler and the four wheeler, which consists of a collection of reusable, more durable, washable carry bags made of cotton or materials like leather. The collection of the carry bags can be enclosed in a box like container and fitted like a toolbox in every vehicle including four wheeler and two wheelers. If the proposed policy is implemented the Vehicles manufacture in future need to fit the VEKIT just like a first aid box. The vehicles in use can make this as an extra fitting.

Based on our proposed recommendation, we tried to find out the possible reduction through an extensive survey. The survey was made based on the following questions to the customers of various shops: (1) awareness to PCB hazards, (2) willingness to reduce the usage of PCB, (3) acceptance to our policy recommendation, (4) readiness to shift from demanding carry bags, if they have a alternate in hand based on our recommended policy implementation.

\section{Outcome of the survey}

The impact of the survey is shown in the Figure 2. Let $n$ represents the no of categories in which the survey is applied, $T$ in Equation (1) represents the total number of buyers came by two wheelers who agreed to the reduction policy, $F$ in Equation (2) represents the total number of buyers came by four wheelers who agreed to the reduction policy, $R$ in Equation (3) represents the average reduction, which can be achieved through the policy reduction on a daily basis. $x_{i}$ represents the no of buyers came by two wheelers who agreed to the reduction policy in each category of the shop and $y_{i}$ represents the no of buyers came by four wheelers who agreed to the reduction policy in each category of the shop.

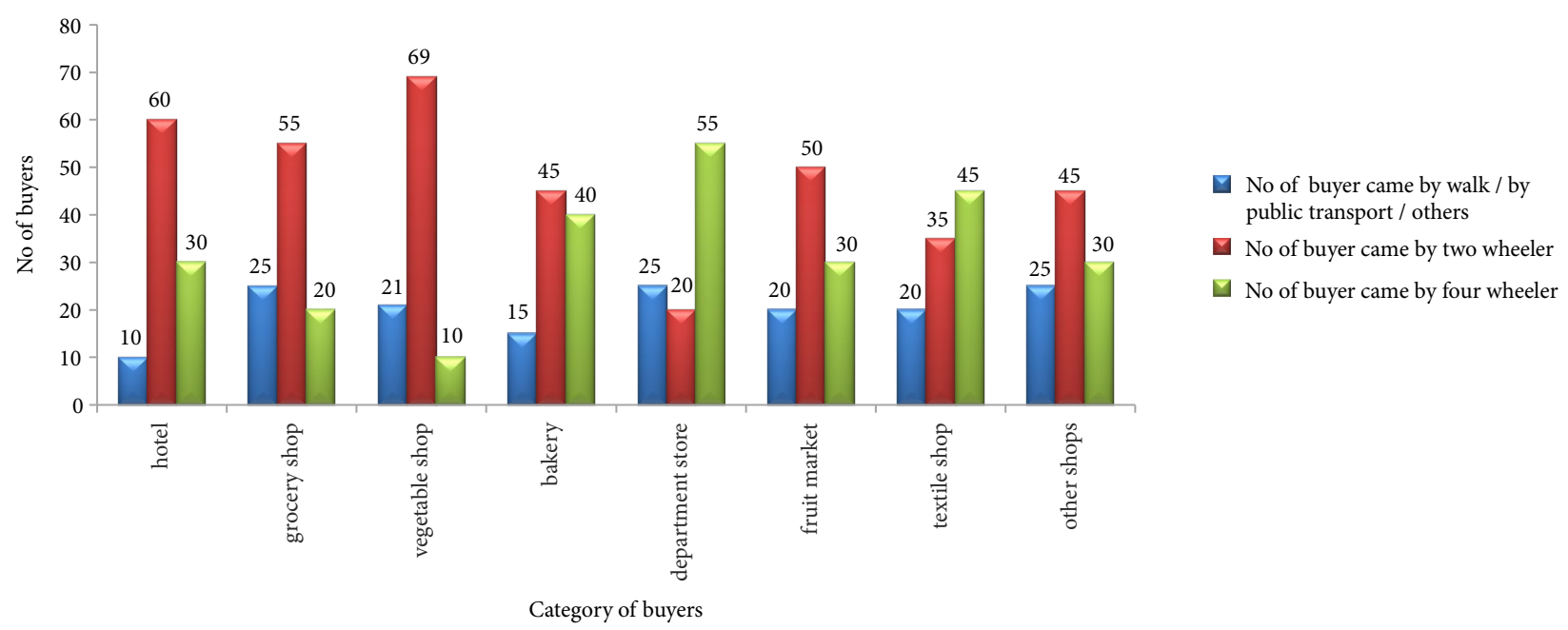

Figure 1. VEKIT research survey showing the percentage of buyers using different transportation 


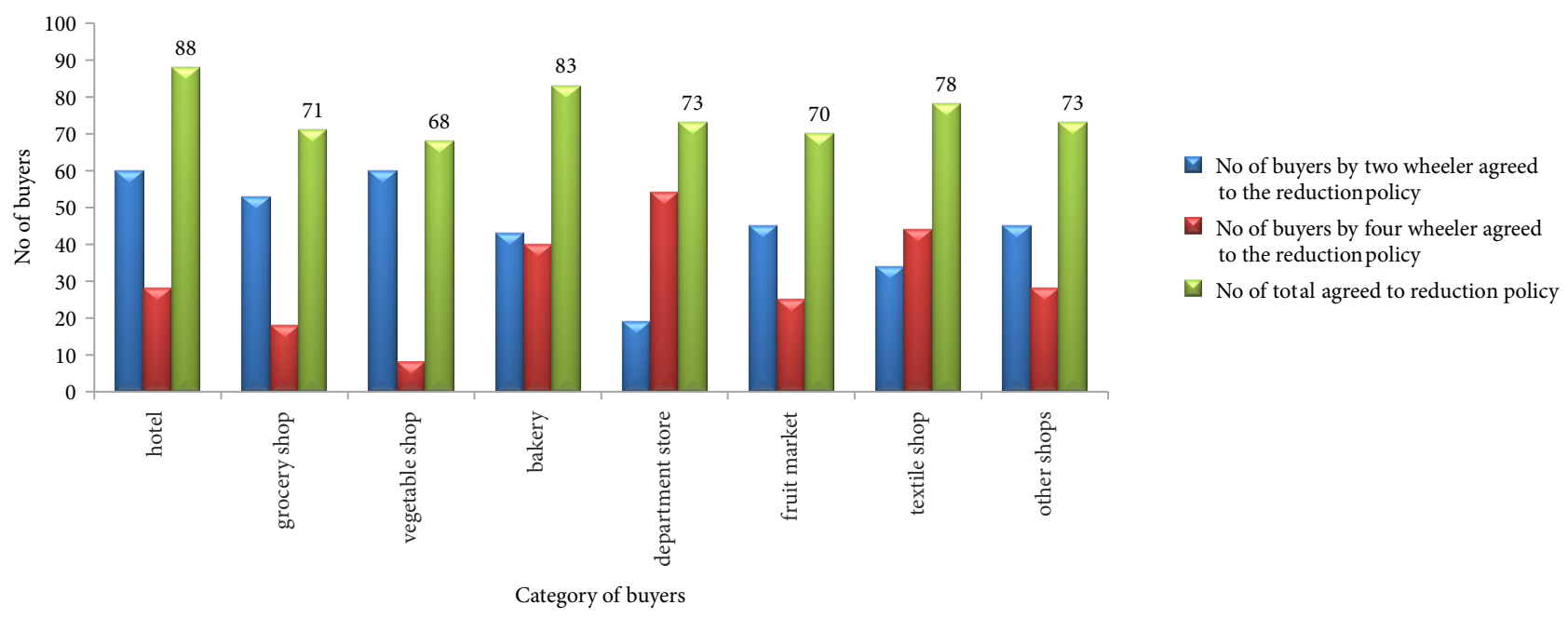

Figure 2. Impact of survey results on VEKIT

$$
\begin{aligned}
& T=\sum_{i=1}^{n} x_{i} ; \\
& F=\sum_{i=1}^{n} y_{i} ; \\
& R=\frac{T+F}{n} .
\end{aligned}
$$

The most interesting finding, we got from the survey is that the customers are very much aware of the hazards of $\mathrm{PCB}$, and are ready to use reusable Bags that are fitted in the two wheelers and four wheelers, if our policy is implemented. This is the basis of the VEKIT methodology, which is used in the design and monitoring scenario.

It also showed a $75.5 \%$ reduction in the usage of $\mathrm{PB}$ on a daily basis. This means that an average reduction from 60 to $70 \%$ in usage of PCB is definitely possible if we are committed to implement our recommended policy based approach.

\section{Other strategies recommended to reduce PCB}

The solution for problems related to $\mathrm{PB}$ and other packaging materials has been handled by many countries around the world using a variety of approaches (EA 2006; BBC 2002; Smith 2004). In some countries like Bangladesh PB manufacturing and usage was banned in 2002. Similar effort has been taken by Hong Kong by implementing an awareness campaign to reduce the $\mathrm{PB}$ usage. The $\mathrm{PB}$ are the main reason for the blockage of water drains, which cause floods in the cities. Due to this Bombay tried to ban the manufacture after floods in 2000 and 2005. In spite of the efforts by the authorities of Bombay city an illicit trading of PB had developed. These facts shows that efforts taken to educate on hazards, levy tax for usage or Banning single usage $\mathrm{PCB}$, are not giving expected reductions as they are not followed which clearly indicates the usefulness of our approach.

\section{Significance of VEKIT approach}

Research presented in the report by EA (2006) supports the importance of our approach. There is a need to develop the practice of using long lasting and reusable bags to reduce environment pollution. The VEKIT approach is based on developing such practice among the people going for shopping using their two wheelers and four wheeler vehicles. The VEKIT monitoring presented in Section 11 is an additional feature to be used with the future VANET infrastructure for ensuring authentication of the vehicles and the working condition of the VEKIT. VEKIT is based on VANET as it has the advantage of mobility when compared to the wireless sensor networks. Even if the policy is made to shift to biodegradable bags (Catchpole 2005), there is only a little gain. The experiments made on the biodegradable bags proved that the plastic does not fully degrade and remains in smaller pieces even after the bacterial action. Hence the usage of VEKIT can reduce the pollution.

\section{Implementation challenges and methods}

The VEKIT, a proposed design with the collection of reusable bags can be fitted, when the vehicles are manufactured using a law. The VEKIT for the vehicles in use can be purchased in the market. The vehicles in use can be inspected to include this container kit like any road inspection by the authorities. The paper proposes an automated inspecting mechanism using VANET infrastructure. There is also a possible end of lifetime for the bags used in the container, and a new kit has to be replaced just like any other spare parts available for a vehicle. Another challenge is the inspection of the vehicles for its authenticity, which can be solved by adding security feature. The methods and degree of strictness to follow are in the hands of the authorities and the governments. The VEKIT is to be installed in the vehicles and can be inspected by the authorities using the VANET infrastructure. 
The VEKIT status message is received from the vehicles once the vehicles passes the region of the VEKIT TU. The status message is used to identify the condition of the VEKIT and the authenticity of the vehicles.

\section{VANETs: an overview}

VANETs are a special type of mobile ad hoc networks (Manvi, Kakkasageri 2008), where vehicles are simulated as mobile nodes. In many parts of the world experiments are made to implement different applications for assisting drivers, controlling and monitoring vehicle traffic using Vehicular Networking (Karagiannis et al. 2011).

VANETs have a full potential to enable the communications among vehicles and between vehicles and RSUs (Eiza et al. 2013). The details of VANET projects were presented in Section 2. In future we expect that each vehicle has a wireless communication facility using one of the many technologies available to provide ad hoc network connectivity. The research and the experiments of VANETs are focused to utilize road safety information among vehicles to prevent accidents. The future vehicles will be equipped with sensors delivering various parameters (Balaanand et al. 2015, 2018; Maram et al. 2019; Anupriya et al. 2018), which will be displayed to the driver or transmitted to a RSU, which is a part of the VANET infrastructure. These parameters are also broadcasted to neighboring vehicles (Zeadally et al. 2012). Apart from
VANET applications focusing on road safety, travel assistance, Internet connectivity, in this work, we have shown that VANET infrastructure could also be used to prevent and reduce environment pollution and act as a vehicle monitoring system for the inspecting authorities.

\subsection{VANET architecture}

The architecture and the components of VANET (Eiza et al. 2013) can be broadly classified into three categories namely V2V, V2RSU and inter-RSU communications.

\subsection{Components of VANET infrastructure}

The VANET infrastructure consists of components like hardware in the vehicle called as OBU and on road-side termed as RSU. The hardware will be equipped with wireless units or radio interfaces to transmit information over short-range using ad hoc networks (Chandler et al. 2010). The RSUs, which are fixed connects the vehicles to the VANET backbone network. The number of RSUs and their position depends on the protocol and the application used.

\section{VEKIT monitoring scenario}

Figure 3 shows the VEKIT monitoring scenario and the use case of the VEKIT and the Figure 4 shows the components of VEKIT. The cars shown in the picture has VEKIT

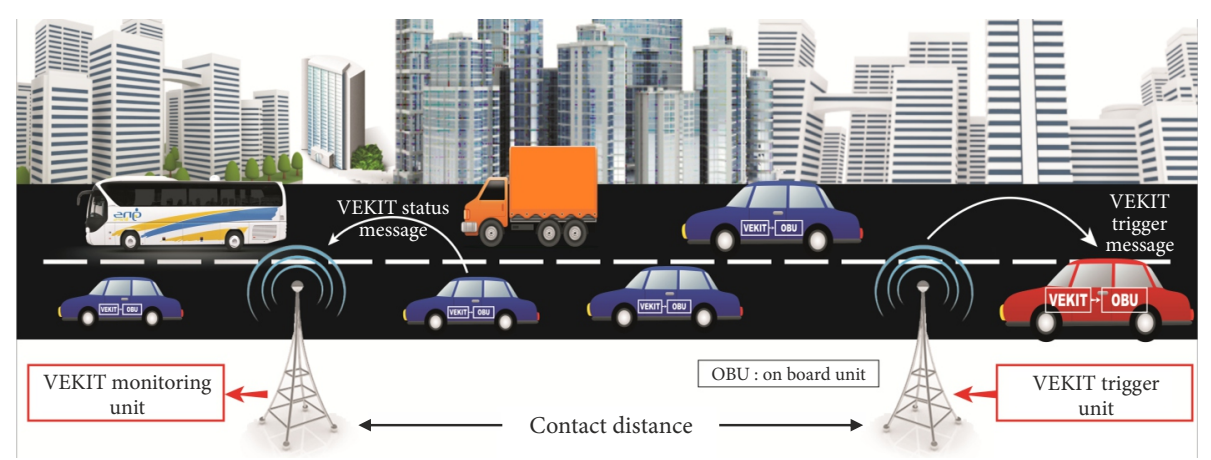

Figure 3. VEKIT monitoring scenario

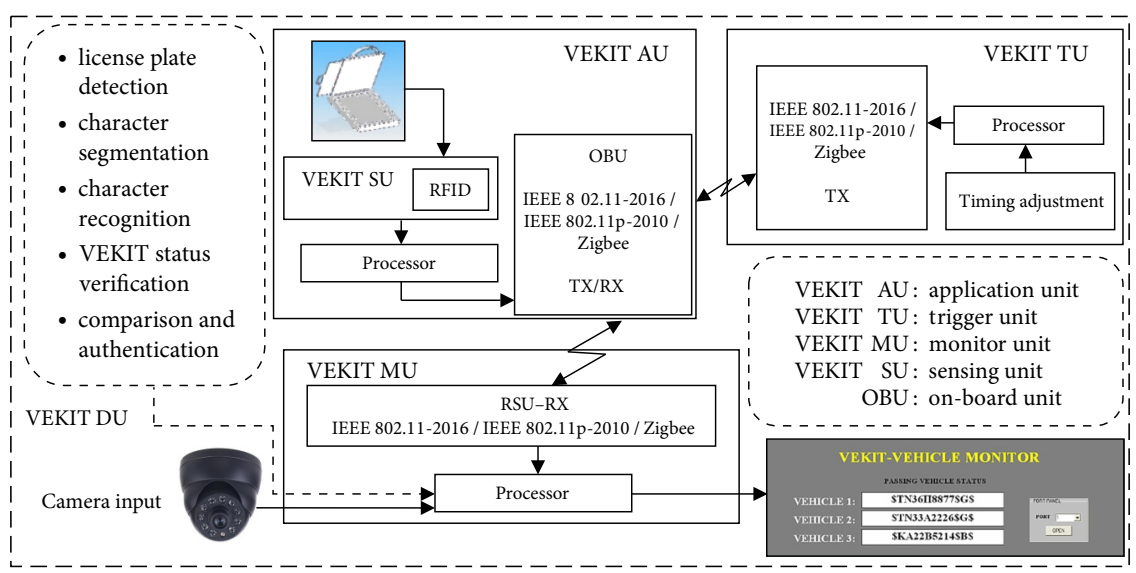

Figure 4. Components of the VEKIT monitoring system 
module connected to the RSU of the VANET infrastructure through the OBU. The VEKIT MU and the VEKIT TU are similar to the RSU of the VANET infrastructure. The presence and the working condition of the VEKIT in the vehicles passing the road can be inspected by the authorities to ensure the usage of VEKIT by all vehicles in the region of interest.

The authorities use the Aadhaar based web Interface in the VEKIT monitoring system to detect if any of the passing vehicles do not have VEKIT installed in it and also the status of the VEKIT. The status of the VEKIT has two possible working condition of the VEKIT as "good" and "bad". The authorities can see the condition of the VEKIT of the vehicles passing them and can take necessary action on the vehicles like stopping the vehicle for filing a case or levy a fine as enforced by the regulation. The VEKIT MU has to be installed in a road where the authorities wish to perform the task of inspecting the vehicles. The VEKIT MU and the VEKIT TU are detachable units that can be installed in a place or a road of interest and can be shifted to another place or road when required. This provision helps the authorities to perform inspection in different roads of a city so that vehicles in all regions can be inspected.

\section{VEKIT monitoring procedure}

The VEKIT monitoring task of the authorities starts with the installation of VEKIT TU in a place $0.5 \mathrm{~km}$ ahead of the VEKIT MU. The VEKIT TU is positioned in a city lamp post or a tall building so that the VEKIT TU trigger message reaches all the vehicles passing the road. The VEKIT TU is a VANET standard transmitter that can send VEKIT trigger message through Wi-Fi, DSRC mode or a zigbee unit. The VEKIT trigger message makes the VEKIT SU active. Once the VEKIT SU is made active, The VEKIT SU starts reading the authentication details, which includes: (1) details of the owner of the vehicle, (2) the registration number of the vehicle stored in the nonvolatile memory of the VEKIT or a smart card, (3) the validity of the insurance of the vehicle, (4) the working condition of the VEKIT as "good" or "bad". The condition "good" indicates that the VEKIT has atleast one reusable bag inside it and is available for the user. These details are transmitted via the OBU to the VEKIT MU through the desired multihop protocol. The multihop mode of signal transmission is useful in preventing the loss of VEKIT data from any single vehicle as the vehicles are moving and will pass the VEKIT MU in a very short interval. The VEKIT MU includes a standard VANET signal RU, a camera, image processing logic, a processor and the VEKIT MU monitoring device.

The method of triggering and receiving the details like Authentication data, status message as explained above is better than periodical transmission method of data from the VEKIT unit as the later method may produce a large number of unnecessary transmissions.

\section{VEKIT system architecture}

The overall VEKIT system architecture shown in the Figure 4. Contain the following functional blocks presented in sub-sections below.

\subsection{VEKIT AU}

The AU is the block carrying the reusable bags stitched with RFID tags. The reusable bags are placed in the VEKIT case as shown in the Figure 5. The bags when taken out for usage in the shops are detected by the RFID reader in the SU. In the same way, it senses the bags when replaced in the VEKIT case after usage.

The AU decides the condition of the VEKIT as "good" when atleast one reusable bag is available for use in the VEKIT. Hence this ensures that one reusable bag is always available with the user going for shopping and there is no need for him to demand for a carry bag. The AU is connected to the OBU. The OBU is a wireless transceiver using any one of standards like Wi-Fi, DSRC or zigbee.

\subsection{VEKIT MU and TU}

The VEKIT MU in Figure 6 consists of wireless transceivers, DU, processor, VEKIT vehicle monitor software. The TU is a detachable separate wireless transceiver that transmits the periodical trigger pulses to the VEKIT AUs installed in moving vehicles.

The VEKIT vehicle monitor software shown in Figure 7 is the front end program that is installed with a laptop or a mobile unit to see the status VEKIT installed in the incoming vehicles. The software recieves the status information from the MU and displays in the screen. The prototype software allows to monitor three vehicles at any particular time and once the vehicles passes the MU, the status of the following vehicles can be viewed.

In this way, the inspecting authorities can have a continouos monitoring procedure without disturbing the flow of vehicles.

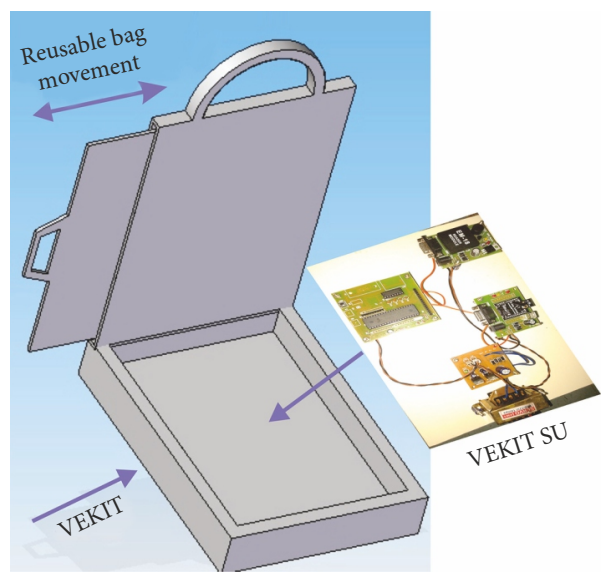

Figure 5. VEKIT prototype model case holding the reusable bag and the $\mathrm{SU}$ 

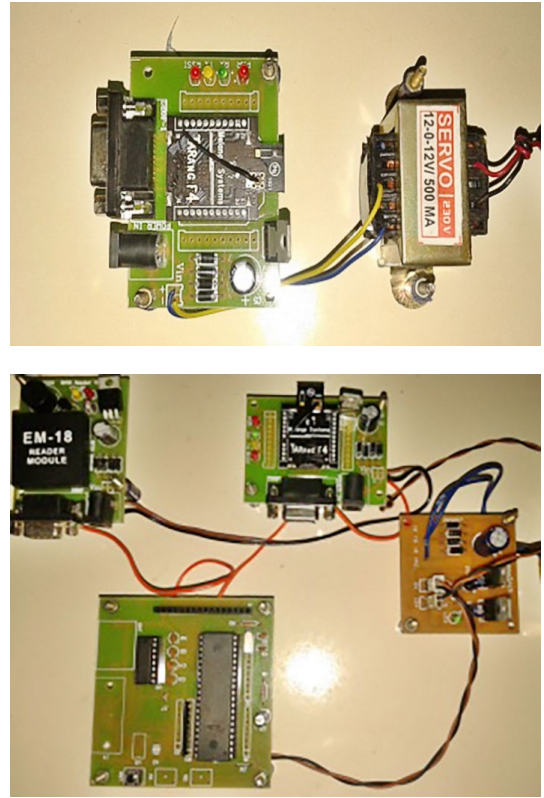

Figure 6. Parts of VEKIT prototype SU and MU

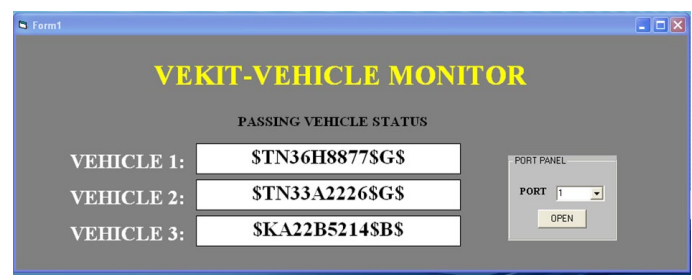

Figure 7. VEKIT front end software screenshot showing VEKIT status

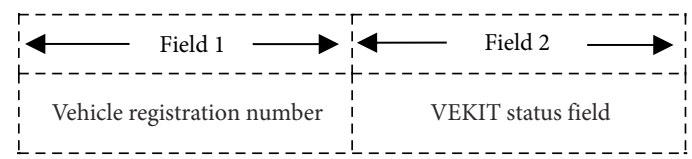

Figure 8. Vehicle status information pattern

The flow of vehicles will be stoppedonly if any of the vehicles with status "bad" is reported by the VEKIT vehicle monitor. The software screenshot shown in the Figure 8 indicates the status of the vehicles under inspection. The passing vehicle status column in the display screen consist of two field as shown in the Figure 8. The prototype gives only two information fields. In addition to these, the following information can be sensed and added in the original VEKIT design: (1) status of the vehicle insurance defining the "validity of the insurance", (2) working condition and expiry of the medical first aid kit, (3) information about the driver like "tiredness grade", "alcohol consumption", etc.

\subsection{VEKIT DU}

The VEKIT DU is used to extract the vehicle registration number from the vehicle license plate. The image of the vehicle license plate is captured using the camera

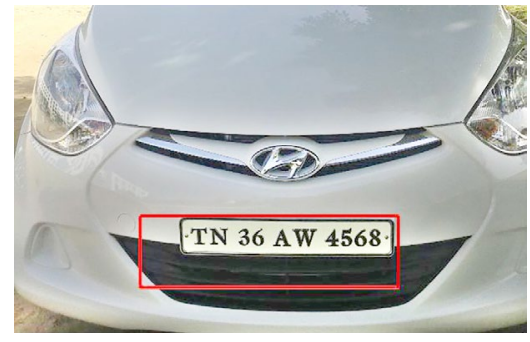

Figure 9. A stage of VEKIT license plate detection

\section{TN 36 AW 4568}

Figure 10. Cropped license plate

in a roughly estimated region of interest and the detection algorithm extracts and provides the vehicle registration number to the MU. Vehicle license plate detection is used automated entry in prepaid parking system, road toll collection, etc. The license plate DU has three major functions namely: (1) license plate detection, (2) character segmentation, (3) character recognition.

Figure 9 shows a stage of VEKIT DU with the detected region of interest marked inside rectangle box.

Figure 10 shows the cropped license plate from the image. The DU sends the recognised number to the MU , which is used to stop the vehicle at the inspection site. Many methods of vehicle license plate detection system are based on edge statistics and morphology (Bai, Liu 2004; Zheng et al. 2005).

The DU of the VEKIT prototype is implemented based on the work by Anagnostopoulos et al. (2006) using sliding concentric windows, connected component analysis and a probabilistic neural network. The detection algorithm based on image segmentation is suitable for the VEKIT monitoring and is also used in automatic parking systems.

\section{Background technologies}

This section covers the potential technologies used in OBUs and RSUs of the VANET infrastructure.

\subsection{Edge}

The GSM technology could be used for the communication between vehicles with just minor changes in a function of special modules. The GPRS could be used for the purpose of transmission and receive, which is currently used for the transmission of data. This technology is highly dependent on the movement speed of a mobile station.

\subsection{Zigbee}

Zigbee technology is a quite new standard based on protocol standard IEEE 802.15.4-2020 and is designed for connecting low-power devices in PAN. Transfer rate reaches from 20 to $250 \mathrm{~kb} / \mathrm{s}$. 


\subsection{DSRC}

DSRC used in VANET is a short to medium range communications that supports $\mathrm{V} 2 \mathrm{~V}$ and V2RSU communications (Zeadally et al. 2012; Harigovindan et al. 2012). DSRC finds many applications in VANET based systems, including V2V safety messages, traffic information collection, toll collection, drive-through payment.

\subsection{WLAN/Wi-Fi standards}

WLAN or Wi-Fi standards are extensively used to create Ad-hoc networks and in VANET due to their low implementation cost, high speed data transfer and their existence in the vehicles for providing internet access. These Consist of several standards like IEEE 802.11a-1999, IEEE 802.11b-1999, etc.

\section{VEKIT performance evaluation}

The VEKIT prototype and the developed trigger algorithm is evaluated using simulation. A simulation model is developed to analyse the performance of the VEKIT scenario using NS2 (https://www.isi.edu/nsnam/ns) software. The simulation model uses a highway scenario with two way road. The vehicles move in opposite directions in each road.

\subsection{Simulation objectives}

The VEKIT simulation is performed to find the following:

- to find the suitability of the data rate that can be achieved using the trigger based algorithm in the mobile conditions of the vehicles with high speed.

- to select the routing protocol, which gives higher PDR and lower end-to-end delay in the trigger based VEKIT implementation.

The simulation parameters selected for the model is listed in the Table.

The Simulation area is chosen to model the inspection area of $2 \mathrm{~km}$ in length and $700 \mathrm{~m}$ wide two way road. The mobility model of the nodes is chosen as random way point, as it models the random behaviour of vehicles. The speed of the mobile nodes is varied between $5 \ldots 50 \mathrm{~m} / \mathrm{s}$, which covers the range of vehicle speeds. Two ray ground is selected as the radio propagation model.

Table. Simulation parameters

\begin{tabular}{|l|l|}
\hline \multicolumn{1}{|c|}{ Parameter } & \multicolumn{1}{c|}{ Value } \\
\hline Simulation area & $2000 \times 700 \mathrm{~m}$ \\
\hline Number of vehicles & 40 \\
\hline Average speed of vehicles & $5 . .50 \mathrm{~m} / \mathrm{s}(18 . .180 \mathrm{~km} / \mathrm{h})$ \\
\hline Mobility model & random way point \\
\hline MAC protocol & 802.11 \\
\hline Routing protocol & T-AODV, T-DSR \\
\hline Radio propagation model & two ray ground \\
\hline
\end{tabular}

\subsection{Routing protocols}

The VEKIT simulation is performed using two famous routing protocols namely AODV and DSR with trigger based implementation (T-AODV, T-DSR). The trigger as seen in the VEKIT scenario is used to start the transmission of VEKIT status data to the MUt installed in the inspection roadway. The AODV algorithm (Perkins et al. 2003) allows dynamic, multihop routing between mobile nodes trying to form and maintain an ad hoc network. The advantage of AODV protocol is that it does not need to maintain the routes to destination nodes that are not active. In DSR protocol (Johnson et al. 2007) route discovery and route maintenance each operate entirely "on demand". It is similar to AODV in forming a route ondemand when a transmitting node requests one. But, it uses source routing instead of using the routing table at each intermediate node. The VEKIT uses the Trigger based protocol for getting the status message from the vehicles. The Simulation presented below helps to select the protocol for a particular application like in VEKIT.

\subsection{Performance metrics}

PDR is obtained by dividing the total number of packets arriving at the destination with the total packets send by all sources. The performance of the network will be better for high PDR.

Average end-to-end delay is the time taken by a packet to route through the network from a source to its destination.

\subsection{Simulation results}

The VEKIT simulation is performed with two routing protocols namely T-AODV and T-DSR by varying the speed of the mobile nodes from 5 to $50 \mathrm{~m} / \mathrm{s}$. The results are presented in the Figures 11 and 12.

The simulation results were used to select the protocol for VEKIT prototype. T-AODV showed better performance in $20 \ldots 40 \mathrm{~m} / \mathrm{s}$. The results show the feasibility of the VEKIT implementation by providing the good packet delivery for the inspection of vehicles while moving in the roads. Various other protocols can also be compared for VEKIT implementation. In this paper, we restricted our discussions with two protocols.

\subsection{Integration of road scenario in simulation}

The simulation is also carried out to investigate the feasibility of the VEKIT Approach using the real traffic scenarios modelled using 2 tools:

- UMO 0.30.0 (https://www.eclipse.org/sumo);

- OMNeT++ 4.6 (https://omnetpp.org).

The VEKIT model is analysed for the 2 types of scenarios with $1 \mathrm{RSU}$ and with 2 RSUs.

The SUMO model shown in Figure 13. is used with $\mathrm{OMNeT}++$ to analyse the performance of IEEE $802.11 \mathrm{p}-2010$ transmitter in the OBU. The transmission uses Inetmanet-2.0 models for the DSRC characteristics. 
The results in Figure 14 show a low latency for the proposed trigger based scenario. This is also investigated with different protocols. The evaluation of the VEKIT performance is made using two scenarios. The scenario 2 with 2 RSUs with one unit placed as a TU showed less delay compared to the scenario 1 with a single RSU. This helps to evaluate the trigger based algorithm used in VEKIT. The platform and the model framework (De Fuentes et al. 2014) can be used as a guide for the network planners to design such interoperable and inter accessible common web interface for VEKIT projects.

\subsection{VEKIT Wi-Fi performance evaluation}

The VEKIT MU is designed to evaluate the status of the VEKIT in "good" or "bad" with the necessary reusable bags in usbale condition. The VEKIT prototype is tested

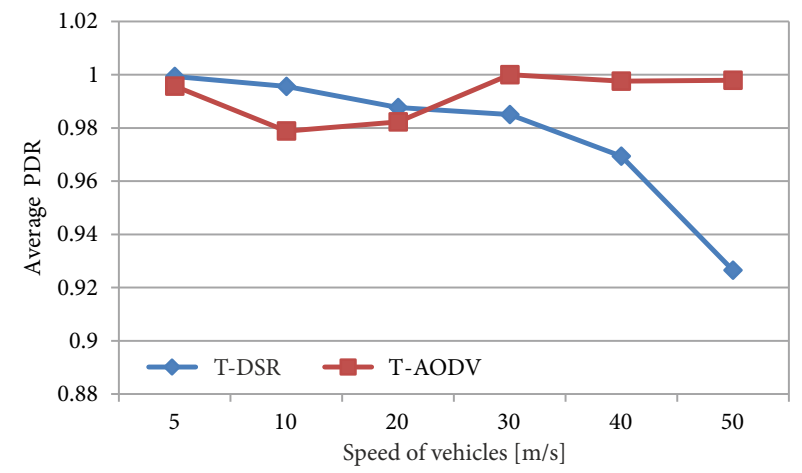

Figure 11. Average PDR for T-DSR and T-AODV

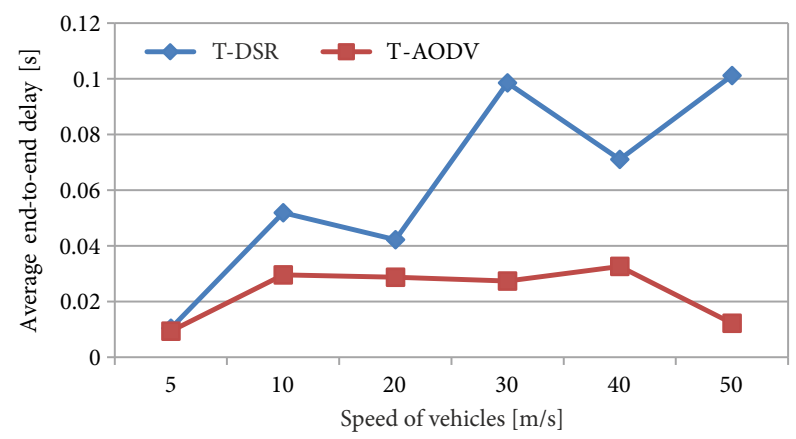

Figure 12. Average end-to-end delay for T-DSR and T-AODV

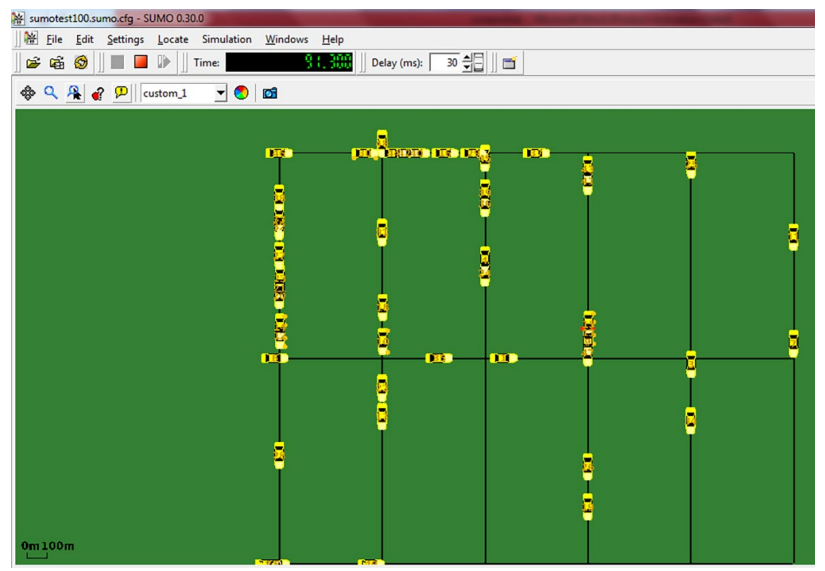

Figure 13. Simulation of real traffic scenario using SUMO with the Wi-Fi transmitter and the receiver in a real time environment. The monitoring software shown in Figure 15 gives the status of VEKIT in the C4 field as "good" denoted by a value 1 or as "bad" denoted by the value 0 . The status in the Figure 14 depicts a "bad" condition of the connected VEKIT. Once the vehicle reaches the monitoring area, the web interface can read the status of the VEKIT, which helps the authority to understand the status of the VEKIT installed in the vehicle.

The advantage of the VEKIT is the implementation methodology, which uses a STEMSEL hardware (https:// stemsel.com/stemsel_automotive_projects.php) that can be programmed in a flow chart like environment as shown in the Figure 16. This method can be applied to any projects involving $\mathrm{Wi}-\mathrm{Fi}$ in automotive applications and VANET scenarios.

\section{VEKIT security and authentication}

The important aspect of VANET project is the security and attacks. The security and authentication is ensured for the VEKIT using the Aadhaar based web Interface. Aadhaar number (Balaanand et al. 2020) is a 12-digit unique number issued by the UIDAI to the citizens of India subsequent to fulfilling the check procedure set by the authority. Any individual, regardless of age and gender, who is residing in India, may apply to get Aadhaar number.

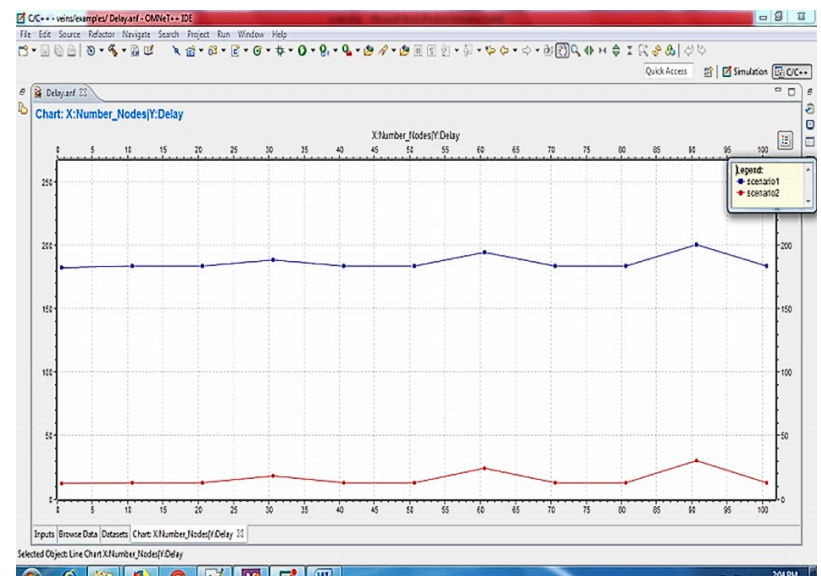

Figure 14. Latency graph for different number of nodes

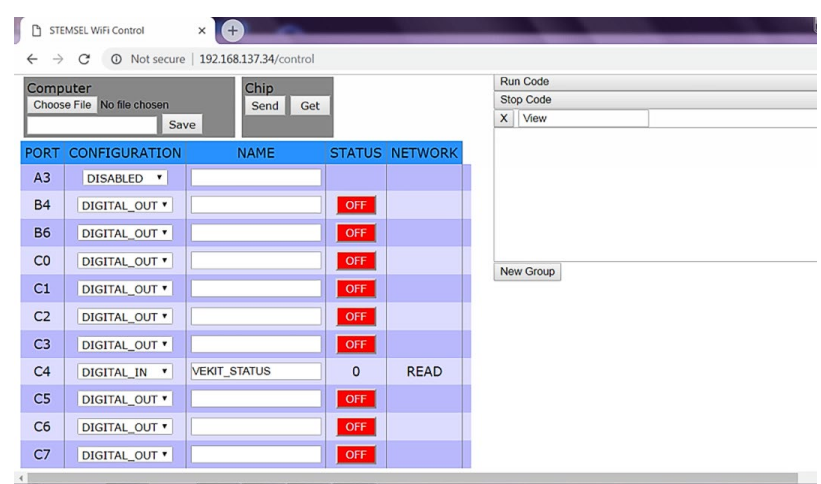

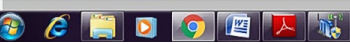

Figure 15. Wi-Fi monitoring software front end 


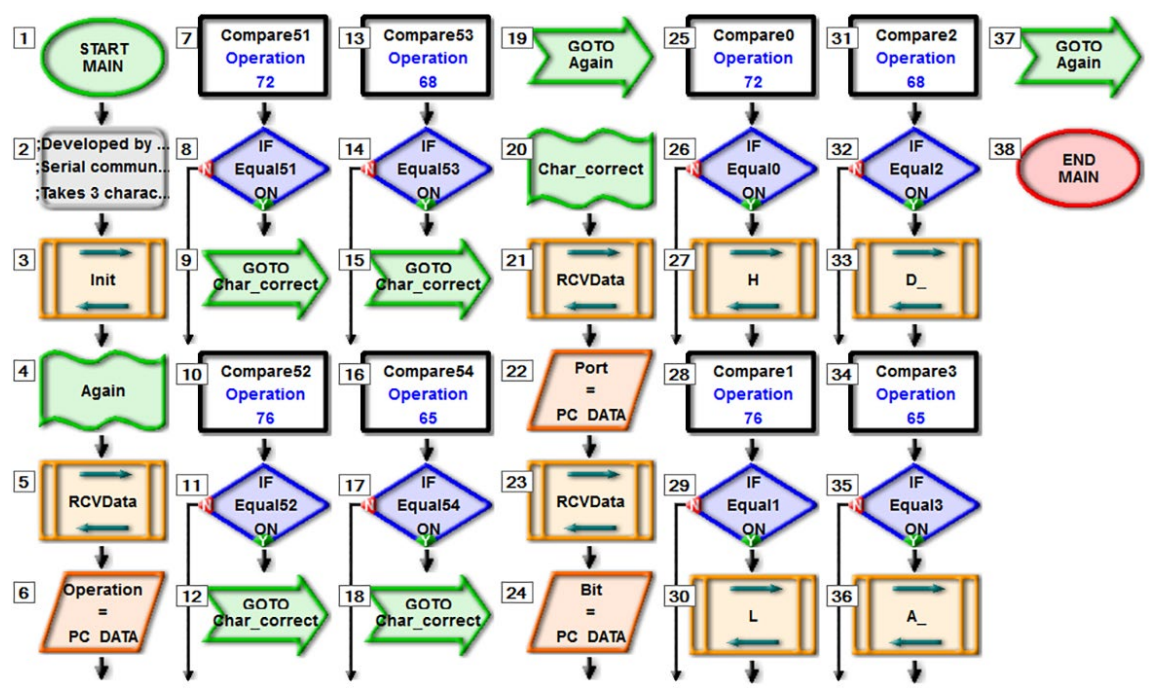

Figure 16. VEKIT Wi-Fi implementation flow chart

Individual willing to get Aadhaar number needs to submit proofs of address, age and biometric data to the enrolment procedure, which is absolutely free of expense. The Aadhaar enrolment process gathers demographic data like name, date of birth, gender, address, mobile number and e-mail ID and the biometric information like ten fingerprints, two iris scans and facial photographs.

\section{Aadhaar based authentication for VEKIT}

The VEKIT security can be enhanced using the digital India initiative - Aadhaar. Aadhaar provides an opportunity to provide security feature to VEKIT by utilising its characters like: (1) uniqueness achieved through the process of demographic and de-duplication, (2) portability, as it can be authenticated online, (3) scalable technology architecture, which helps to handle 100 million authentications a day, (4) open source technologies, structured to address scalability in a vendor neutral manner.

Aadhaar authentication is used to provide a digital, online identity platform so that the identity of Aadhaar number holders can be checked quickly anytime, anyplace. If the Aadhaar enrolment and authentication is linked with the VEKIT in the vehicles the security can be enhanced.

UIDAI offers Aadhaar based authentication as a facility that can be utilised by requesting organisations like government, public and private entities, agencies. This facility can be utilised by various organistatons to provide services to their customers. The Aadhaar card based authentication is used at present for services like LPG subsidy transfer, opening a bank account using Aadhaar as a single sufficient document, getting a passport, etc. The Figure 17 shows the proposed security architecture for the VEKIT system. The vehicle owners are required to enrol for the Aadhaar linking through the authorised user Agencies. The VEKIT monitoring is done in the roads with the VEKIT monitor using the web interface provided in the digital gadgets like a mobile device by the monitoring authorities of the traffic department.

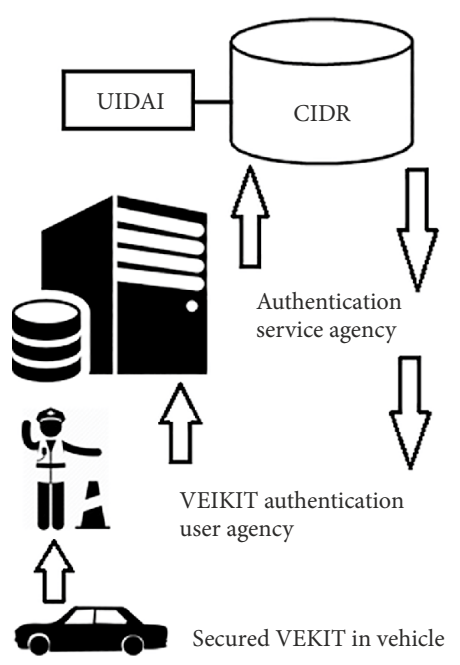

Figure 17. Proposed VEKIT security architecture

\section{Conclusions and future directions}

VEKIT is a novel system suggested for preventing environment pollution using the future VANET projects. VEKIT adds value to the VANET projects in the early stage of implementation. As the added value of one vehicle deployed with VANET OBU to introduce direct communication between vehicles and communication between vehicle and RSU depends on the total number of vehicles having OBU. In case of VEKIT project, the VANET adds value to help prevent environment pollution. VEKIT is different from various projects suggested in the area of transport Information technology and connected vehicle based applications. This VEKIT based VANET deployment will be more appropriate in near future when there is a shift to electric vehicles or hybrid vehicles comes to practice. In that case there will be a reason for the authorities to introduce a new unit mandatorily in every new vehicle that is manufactured and sold in the market. 
The Union Ministry of Environment (PTI 2015) has reported that atleast $30 \mathrm{~kg}$ of plastic can be found from the stomach of every cow or buffalo, which dies in India highlighting the severity of the problem. The report from the ministry expressed the concern over the difficulty in banning the plastic based carry bags and in implementing the laws. The VEKIT prototype presented in this paper is implemented based on the outcome of VEKIT policy based approach presented in Section 6. The VEKIT prototype helps to reduce the environment pollution caused by the usage of PCB. The paper presented the ways and technologies behind the VEKIT project. Hence we conclude by saying that our VEKIT policy based approach as shown in Figure 2, of fitting VEKIT having a collection of reusable bags in the vehicles, can cause a $60 . .75 \%$ reduction in the PCB usage. Hence our approach has the full potential to resolve an issue, which is creating a threat to the whole earth and the mankind. The work presented in the VEKIT project is a novel idea to reduce the environment pollution. The paper addressed the need for the VEKIT for reducing the environment pollution. The paper focused more on the justification of the need of the proposed VEKIT. The details of the Hardware Implementation using Wi-Fi and the VEKIT node architecture will be presented in our future work. While it addressed some of the necessary security concerns like Identification, authenticity using Aadhaar based web interface; there is a much scope for other security feature additions and the VEKIT design optimisations to improve the monitoring web interface, which can be addressed through further research in this area.

\section{References}

Ahmed-Zaid, F.; Bai, F.; Bai, S.; Basnayake, C.; Bellur, B.; Brovold, S.; Brown, G.; Caminiti, L.; Cunningham, D.; Elzein, H.; Hong, K.; Ivan, J.; Jiang, D.; Kenney, J.; Krishnan, H.; Lovell, J.; Maile, M.; Masselink, D.; McGlohon, E.; Mudalige, P.; Popovic, Z.; Rai, V.; Stinnett, J.; Tellis, L.; Tirey, K.; VanSickle, S. 2011. Vehicle Safety Communications - Applications (VSCA) Final Report. Report No DOT HS 811 492A. National Highway Traffic Safety Administration, US Department of Transportation, Washington, DC, US. 102 p. Available from Internet:

https://www.nhtsa.gov/sites/nhtsa.dot.gov/files/811492a.pdf

Al-Sultan, S.; Al-Doori, M.; Al-Bayatti, A. H.; Zedan, H. 2014. A comprehensive survey on vehicular ad hoc network, Journal of Network and Computer Applications 37: 380-392.

https://doi.org/10.1016/j.jnca.2013.02.036

Anagnostopoulos, C. N. E.; Anagnostopoulos, I. E.; Loumos, V.; Kayafas, E. 2006. A license plate-recognition algorithm for intelligent transportation system applications, IEEE Transactions on Intelligent Transportation Systems 7(3): 377-392. https://doi.org/10.1109/TITS.2006.880641

Anupriya, K.; Gayathri, R.; Balaanand, M.; Sivaparthipan, C. B. 2018. Eshopping scam identification using machine learning, in 2018 International Conference on Soft-Computing and Network Security (ICSNS), 14-16 February 2018, Coimbatore, India, 1-7. https://doi.org/10.1109/ICSNS.2018.8573687

Attinger, M. 2011. The Problem with Indestructable Plastic Bags. Available from Internet:

https://hubpages.com/food/The-Problem-With-Plastic-Bags
Bai, H.; Liu, C. 2004. A hybrid license plate extraction method based on edge statistics and morphology, in ICPR 2004: Proceedings of the 17th International Conference on Pattern Recognition, 23-26 August 2004, Cambridge, UK, 2: 831-834. https://doi.org/10.1109/ICPR.2004.1334387

Balaanand, M.; Karthikeyan, N.; Karthik, S. 2020. Designing a framework for communal software: based on the assessment using relation modelling, International Journal of Parallel Programming 48(2): 329-343. https://doi.org/10.1007/s10766-018-0598-2

Balaanand, M.; Karthikeyan, N.; Karthick, S.; Sivaparthipan, C. B. 2018. Demonetization: a visual exploration and pattern identification of people opinion on tweets, in 2018 International Conference on Soft-Computing and Network Security (ICSNS), 14-16 February 2018, Coimbatore, India, 1-7.

https://doi.org/10.1109/ICSNS.2018.8573616

Balaanand, M.; Sowmipriya, R.; Sivaranjani, S.; Sankari, S. 2015. Identifying fake user's in social networks using non verbal behavior, International Journal of Technology and Engineering System 7(2): 157-161.

BBC. 2002. Planet Earth's New Nemesis?, BBC News, 8 May 2002. Available from Internet: http://news.bbc.co.uk/2/hi/ uk_news/1974750.stm

Catchpole, H. 2005. The Indestructibles, $A B C, 27$ January 2005. Available from Internet: https://www.abc.net.au/science/articles/2005/01/27/2839596.htm

Chandler, B.; Beasley, K.; Rephlo, J. 2010. National Evaluation of the SafeTrip-21 Initiative: I-95 Corridor Coalition Test Bed Final Evaluation Report, North Carolina Deployment of Portable Traffic-Monitoring Devices. Contract No DTFH6106-D-00005. Federal Highway Administration, US Department of Transportation, Washington, DC, US. 26 p. Available from Internet: https://rosap.ntl.bts.gov/view/dot/4008

Cronin, B. 2015. Connected Vehicle Benefits. Intelligent Transportations Systems Joint Program Office (ITS JPO), US Department of Transportation, Washington, DC, US. 2 p. Available from Internet: https://www.its.dot.gov/factsheets/pdf/ConnectedVehicleBenefits.pdf

De Fuentes, J. M.; González-Manzano, L.; González-Tablas, A. I.; Blasco, J. 2014. Security models in vehicular ad-hoc networks: a survey, IETE Technical Review 31(1): 47-64. https://doi.org/10.1080/02564602.2014.890844

EA. 2006. Plastic Shopping Bags - Analysis of Levies and Environmental Impacts: Final Report. Department of the Environment and Heritage, Environment Australia (EA). 102 p. Available from Internet: http://www.greenbag.com.au/ UserFiles/AU_analysis.pdf

Eiza, M. H.; Ni, Q.; Owens, T.; Min, G. 2013. Investigation of routing reliability of vehicular ad hoc network, EURASIP Journal on Wireless Communications and Networking 2013: 179. https://doi.org/10.1186/1687-1499-2013-179

Harigovindan, V. P.; Babu, A. V.; Jacob, L. 2012. Ensuring fair access in IEEE $802.11 \mathrm{p}$-based vehicle-to-infrastructure networks, EURASIP Journal on Wireless Communications and Networking 2012: 168. https://doi.org/10.1186/1687-14992012-168

IEEE 802.11-2016. IEEE Standard for Information technology Telecommunications and Information Exchange between Systems Local and Metropolitan Area Networks - Specific Requirements - Part 11: Wireless LAN Medium Access Control (MAC) and Physical Layer (PHY) Specifications.

IEEE 802.11a-1999. IEEE Standard for Telecommunications and Information Exchange between Systems - LAN/MAN Specific Requirements - Part 11: Wireless Medium Access Control 
(MAC) and Physical Layer (PHY) Specifications: High Speed Physical Layer in the $5 \mathrm{GHz}$ Band.

IEEE 802.11b-1999. IEEE Standard for Information Technology - Telecommunications and Information Exchange between Systems - Local and Metropolitan Networks - Specific Requirements - Part 11: Wireless LAN Medium Access Control (MAC) and Physical Layer (PHY) Specifications: Higher Speed Physical Layer (PHY) Extension in the $2.4 \mathrm{GHz}$ Band.

IEEE 802.11p-2010. IEEE Standard for Information Technology - Local and Metropolitan Area Networks - Specific Requirements - Part 11: Wireless LAN Medium Access Control (MAC) and Physical Layer (PHY) Specifications Amendment 6: Wireless Access in Vehicular Environments.

IEEE 802.15.4-2020. IEEE Standard for Low-Rate Wireless Networks. https://doi.org/10.1109/IEEESTD.2020.9144691

Johnson, D.; Hu, Y.; Maltz, D. 2007. The Dynamic Source Routing Protocol (DSR) for Mobile Ad Hoc Networks for IPv4. 107 p. Available from Internet: https://tools.ietf.org/pdf/rfc4728.pdf

Kalpana, P. 2010. Plastic Bags-An Environmental Hazard. Press Information Bureau, Government of India. Available from Internet: http://pibmumbai.gov.in

Karagiannis, G.; Altintas, O.; Ekici, E.; Heijenk, G.; Jarupan, B.; Lin, K.; Weil, T. 2011. Vehicular networking: a survey and tutorial on requirements, architectures, challenges, standards and solutions, IEEE Communications Surveys \& Tutorials 13(4): 584-616. https://doi.org/10.1109/SURV.2011.061411.00019

KWT. 2016. Single Use Carrier Bag Charge: Bags of Opportunity to Make a Positive Impact. Keep Wales Tidy (KWT), Wales, UK. Available from Internet: https://www.keepwalestidy.cym$\mathrm{ru} /$ news/single-use-carrier-bag-charge-bags-of-opportunityto-make-a-positive-impact

Manvi, S. S.; Kakkasageri, M. S. 2008. Issues in mobile ad hoc networks for vehicular communication, IETE Technical Review 25(2): 59-72.

Maram, B.; Gnanasekar, J. M.; Manogaran, G.; Balaanand, M. 2019. Intelligent security algorithm for UNICODE data privacy and security in IOT, Service Oriented Computing and Applications 13(1): 3-15. https://doi.org/10.1007/s11761-0180249-X

Perkins, C.; Belding-Royer, E.; Das, S. 2003. Ad Hoc On-Demand Distance Vector (AODV) Routing. 37 p. Available from Internet: https://tools.ietf.org/pdf/rfc3561.pdf

PTI. 2015. Javadekar says every dead cow in India contains $30 \mathrm{~kg}$ plastic, The Hindu, 28 March 2015. Available from Internet: https://www.thehindu.com/news/national/javadekarsays-every-dead-cow-in-india-contains-30kg-plastic/article7043236.ece

Smith, S. 2004. Plastic Bags. Briefing Paper No 5/04. New South Wales Parliament, Australia. 29 p. Available from Internet: https://www.parliament.nsw.gov.au/researchpapers/Documents/plastic-bags/05-04.pdf

Zeadally, S.; Hunt, R.; Chen, Y.-S.; Irwin, A.; Hassan, A. 2012. Vehicular ad hoc networks (VANETS): status, results, and challenges, Telecommunication Systems 50(4): 217-241. https://doi.org/10.1007/s11235-010-9400-5

Zheng, D.; Zhao, Y.; Wang, J. 2005. An efficient method of license plate location, Pattern Recognition Letters 26(15): 2431-2438. https://doi.org/10.1016/j.patrec.2005.04.014 